\title{
How does laparascopic lateral suspension affect the quality of life (QOL) and sexual activity?
}

\begin{abstract}
Background: The affect of Laparascopic Lateral Suspension on quality of life and sexual activity in POP patients.

Objective: This study aimed to investigate the quality of life (QOL) and sexual activity after Laparascopic Lateral Suspension (LLS) for pelvic organ prolapse (POP) in Turkish women. The practical success rate and complications related surgery were also analyzed.

Study design: Prolapse Quality of Life Questionnaire (P-QOL) was used for investigate QOL in study group. Female Sexual Function Index (FSFI) was wielded for evaluating sexual function. Both tests were asked before the surgery and 12 months later. The results were recorded and retrospectively analyzed.
\end{abstract}

Results: 237 patients were included in this study. Every P-QOL parameter was markedly recovered postoperatively. The complications detected after the surgery were; side pain $13(5.5 \%)$, POP recurrence $4(1.6 \%)$, de novo stress urinary incontinence $2(0.8 \%)$, mesh erosion $1(0.4 \%)$, and de novo dyspareunia $1(0.4 \%)$.Cause of haven't got any partner, 124 of the 237 patients $(52.3 \%)$ reject to finish the FSFI. The rest 113 patients fulfilled the FSFI before and 79 patients completed the FSFI after the surgery. While $13(5.4 \%)$ were sexually active before the surgery, $18(7.5 \%)$ were sexually active after the surgery. The significantly increased domain after surgery was sexual function score with arousal.

Conclusion: In this study, low frequency of sexual intercourse was found in Turkish POP population before and also after the operation. However, LLS technic proved fine QOL resuts with a high success rate. Its complications rate is also low. LLS is safe and effective for both sexually active and inactive POP patients.

Keywords: pelvic organ prolapse, quality of life, uterine suspension
Volume II Issue I - 2020

\section{Adeviye Elçi Atılgan \\ Assistant Professor, Faculty of Medicine, Department of \\ Obstetry and Gynaecology, İstanbul Medipol University, Turkey}

Correspondence: Adeviye Elçi Atılgan, Faculty of Medicine, Department of Obstetry and Gynaecology, Bağclar, İstanbul Medipol University, Istanbul, Turkey, Tel 905057553783. Email dradevye@gmail.com

Received: November I5, 2019 | Published: January 29, 2020
Abbreviations: QOL, quality of life; LLS, laparascopic lateral suspension; POP, pelvic organ prolapse; P-QOL, prolapse quality of life questionnaire; FSFI, female sexual function index; POP, pelvic organ prolapse; POP-Q, pelvic organ prolapse quantification; TVT, tension-free vaginal tape

\section{Introduction}

Pelvic organ prolapse (POP) in particular the postmenopausal group is a general pelvic floor deformation among women. ${ }^{1}$ In general, the wide prevalence of POP ranges from $2.9 \%$ to $50 \%{ }^{1-3}$ and the lifetime risk of surgery is $11-20 \%{ }^{1,4}$ Because bowel, lower urinary tract, and sexual problems accompany POP, it has a significant negative effect on quality of life (QOL). Although mesh extrusion, pelvic pain, dyspareunia, organ perforation, lower urinary tract symptoms and POP.

\section{Manuscript}

recurrence are common surgical complications; many surgical procedures for repairing POP have been developing day by day for improve the patient's QOL. ${ }^{5}$ However, there is little outcome about the results of surgery for POP and patients' QOL with their sexual activity. The aim of this study was to anlayze QOL of Turkish POP patients with the sexual activity before and 12 months after LLS surgery. The success rate and complications rate were also searched.

\section{Methods}

This study was designed as a retrospective study. All patients who underwent LLS surgery for symptomatic POP from May 2015 to March 2017 and who were followed up for 12 months were included the study. All patients provided written informed consent before surgery. The study was conducted in accordance with the Good Clinical Practice guidelines and the principles of the Declaration of Helsinki after being approved by the Necmettin Erbakan University ethics committee.

Initially, all of the patients were examined at lithotomy position, and POP was staged according to the Pelvic Organ Prolapse Quantification (POP-Q) system. ${ }^{6}$ Questionnaires and physical examinations were performed both before and 12 months after the surgery. LLS surgery was performed as described previously by Dubuisson et al. ${ }^{7}$ The technic is fixing a T-shaped polypropylene mesh with a nonabsorbable suture over the vesicovaginal fasia, then the subperitoneal passing of the each lateral arm of the mesh in the lateral abdominal parietal peritoneal wall, at last leaving the skin above the iliac crest, in a place without risks of vascular, nerve, bowel injuries. ${ }^{7}$ We cut a polypropylene mesh according to the anotomy of the patients properly. Postoperative complications, including lower urinary tract symptoms, pain, mesh extrusion, dyspareunia, and the subjective recurrence of POP were evaluated. Presence of POP-Q stage II or over prolapse was defined as objective recurrence of POP. 
To evaluate sexual function, we used the Female Sexual Function Index (FSFI) ${ }^{8}$ and we used Turkish-translated P-QOL questionnaire for a complete understanding of questionaire. The FSFI is a 19-item questionnaire with a total score ranging from 2 to 36 (a higher score represents better sexual function). ${ }^{9}$ The P-QOL is scored from 0 to 100 , with a higher score showing a greater negative impact on POPrelated QOL. ${ }^{10}$ For statistical analysis, Fisher's exact test was used to evaluate the changes in the FSFI response rate, and the Wilcoxon signed rank test was used to assess the changes in the patients' FSFI and P-QOL scores from before to 12 months after surgery.

\section{Results}

In tis study, 237 patients in total underwent gynaecologic examination and answered the P-QOL questionnaire before and then opereted for symptomatic POP with LLS technic. They were followed for 12 months after the surgery (Table 1). Their median age was 65.5 (range: 47-84) years, their median body mass index was 27.3 (range: 18.6-36.1) and their median parity was 2 (range: $0-4$ ). Twenty-five patients $(10.5 \%)$ had hysterectomy before, and 12 patients $(10.5 \%)$ had sexuall active life according to the FSFI questionnaire. The majority of the study group; $93.4 \%$ of the patients $(n=220)$ had stage III or over POP. A total of 91 patients $(38.3 \%)$ underwent anterior colporrhaphy for cystocele, 5 patients $(2.1 \%)$ underwent posterior colporraphy for rectocele, 72 patients $(30.3 \%)$ underwent anterior and posterior colporrhapy for cystocele and rectocele. 12 patients (5.0\%) had symptomatic vault prolapse and underwent Laparascopic Lateral Colposuspansion. Hysterectomy alone or concomitant was not performed in this study group. However, concomitant mid-urethral sling surgery (tension-free vaginal tape-TVT) was performed in 92 patients with symptomatic Stress Urinary incontinance(SUI) (38.8\%). In 4 patients POP recurrence was occured after the LLS. Objective POP recurrence occurred in 2 patients $(0.8 \%)$, and one patient $(0.4 \%)$ underwent reoperation. 2 patients expressed subjective recurrence but there was no recurrence when we examine them. The following postoperative complications observed: mesh extrusion was detected in 2 patients $(0.84 \%)$ (1 patient achieved remission after local hormone replacement therapy, and one patient was managed surgically under local anesthesia). These two patients had vault prolapse. De novo SUI occurred in 13 patients $(5.5 \%) 2$ months later and $5(2.1 \%)$ patients required mid-urethral sling (TVT) operation after 3 months. De novo dyspareunia occurred in one patient $(0.4 \%)$ without mesh extrusion, while the remission of dyspareunia was detected in two patient $(0.8 \%)$ after laparascopic lateral colposüspantion surgery. Inguinal pain was noted in 16 patients $(6.7 \%)$, but it was resolved within 2 months. Table 2 shows prominently improved each PQOL domain scores after LLS surgery.

Table I Patient characteristics $(n=237)$

\begin{tabular}{ll}
\hline Age, years & $65.5(47-84)$ \\
Body mass index & $27.3(18.6-36.1)$ \\
Parity & $2(0-4)$ \\
History of hysterectomy & $25(10.5 \%)$ \\
Sexually active & $12(10.5 \%)$ \\
POP-Q stage & \\
Stage II & $14(6.5 \%)$ \\
Stage III & $174(74.5 \%)$ \\
Stage IV & $46(18.9 \%)$ \\
\hline
\end{tabular}

Data are presented as median (range) or $\mathrm{n}(\%)$; POP-Q, pelvic organ prolapse quantification
Table 2 P-QOL domain scores obtained before and after LLS surgery

\begin{tabular}{llll}
\hline Domains & Before LLS & After LLS & P-value \\
\hline General health & $55.0(0-100)$ & $26.0(0-75)$ & $<0.001$ \\
Prolapse impact & $68.6(0-100)$ & $0(0-66.79$ & $<0.001$ \\
Role limitation & $49.2(0-100)$ & $0(0-83.3)$ & $<0.001$ \\
Physical & $45.0(0-100)$ & $0(0-66.7)$ & $<0.001$ \\
limitations & & & \\
Social limitations & $24.6(0-100)$ & $0(0-66.7)$ & $<0.001$ \\
Personal & $0(0-100)$ & $0(0-33.3)$ & $<0.005$ \\
relationships & & & $<0.001$ \\
Emotions & $43.3(0-88.9)$ & $0(0-66.7)$ & $<0.001$ \\
Sleep/energy & $18.6(0-100)$ & $0(0-33.1)$ & $<0.001$ \\
Severity measures & $36.4(0-75)$ & $0(0-83.3)$ & \\
\hline
\end{tabular}

As seen, the median scores for all domains except the general health domain were zero. It means that the lowest degree of POP symptominduced QOL impairment after surgery. The response rates of the FSFI questionnaire before and at 12 months after surgery were seen in Table 3. Before the LLS surgery, 125 (52.7\%) rejected to complete the FSFI because they were not sexually active. Although 112 patients $(47.7 \%)$ completed the FSFI, 99 patients $(41.7 \%)$ were not sexually active, so only 13 patients $(5.4 \%)$ had regular sexual activity. After the LLS, the number of women who refused to complete the FSFI (because they were not sexually active) increased significantly to 156 $(65.8 \%)$. Among the 81 patients $(34.1 \%)$ who responded to the FSFI after 12 months, $18(7.5 \%)$ were sexually active. Before and after the LLS surgery, the sexuel active patients rate was very low and there was no pointed difference between them $(\mathrm{P}=0.8429)$.

Table 3 Response rate for the FSFI questionnaire $(n=237)$

\begin{tabular}{llll}
\hline Variables & Before LLS & After LLS & P-value \\
\hline No response (no sex life) & I25(52.7\%) & $156(65.8 \%)$ & \\
Responded, not sexually active & $99(41.7 \%)$ & $63(26.5 \%)$ & 0.8429 \\
Responded, sexually active & $13(5.4 \%)$ & $18(7.5 \%)$ & \\
\hline
\end{tabular}

Data are presented as n (\%); FSFI, female sexual function ındex; LLS, laparascopic lateral suspention

Table 4 shows the FSFI scores. 61 patients completed the FSFI scores both before and after LLS surgery. While the score for arousal and lubrication was significantly enhanced after surgery $(\mathrm{P}=0.045)$, the scores for satisfaction, pain, orgasm and desire did not change after the LLS surgery. The patients' all sexual function got better significantly after the surgery $(\mathrm{P}=0.014) .13$ patients $(5.4 \%)$ who were sexually active before surgery, 5 patients were added this group after the operation and 18 patients $(7.5 \%)$ were sexual active after surgery. 
Table 4 FSFI scores before and after LLS $(n=6 I)$

\begin{tabular}{llll}
\hline Variables & Before LLS & After LLS & P-value \\
\hline Desire & $1.2(1.2-4.2)$ & $1.2(1.2-3.8)$ & 0.074 \\
Arousal & $0.3(0-4.5)$ & $0.3(0-3.6)$ & 0.045 \\
Lubrication & $0(0-6)$ & $0(0-6)$ & 0.078 \\
Orgasm & $0(0-6)$ & $0(0-5.8)$ & 0.511 \\
Satisfaction & $2.4(0.8-6)$ & $0(0.8-5)$ & 0.131 \\
Pain & $0(0-6)$ & $0(0-8)$ & 0.79 \\
Total & $4.2(3.6-29.2)$ & $4.4(3.6-29.2)$ & 0.014
\end{tabular}

Data are presented as median (range); FSFI, female sexual function ındex; LLS, laparascopic lateral suspansion

\section{Discussion}

In the present study, significant postoperative improvements in each P-QOL subject after 12 months of LLS surgery and very low incidence of objective recurrence rate $(0.8 \%)$ indicate that this surgical technique can be used more widespreadly in symptomatic POP patients. Anatomic cure was described according to the POP-Q sites; $\mathrm{Ba}, \mathrm{C}$ and $\mathrm{Bp}$ as less than $-1 \mathrm{~cm}$ at any point during followup. Among the 4 patients who express recurrence, only two patients $(0.4 \%)$ had objective recurrence and one of them $(0.8 \%)$ underwent reoperations within 6 months. Takahashi reported reoperation rate for recurrence of POP was $2.3 \% .{ }^{11}$ This present study result is very low according to the data in the literatüre. While subjective and objective recurrence rates of $7-14 \%$ and $23-41 \%$ were reported in a Cochrane meta-analysis, surgical retreatment rates are reported between 5.6 and $19 \%(13,4)$. So, the POP recurrence rate is higher than treatment rates.

The median scores of the P-QOL questionnaire, excluding the general health field, were zero, indicating that more than half of the patients had no complaints related to POP symptoms after LLS surgery. When there are many factors other than POP-related QOL that affect the general health field, this is the least affected parameter directly from POP symptoms.Postoperatif dyspareunia is one of the most common complication of POP surgery. In this study, de novo dyspareunia occurred in one patient $(0.4 \%)$ without mesh exposure, while the remission of dyspareunia was detected in two patient $(0.8 \%)$ after laparascopic lateral süspansion surgery. The exact detection of changes in the sexual function of patients after POP surgery is very difficult, since the sexual activity of the patients will be affected by postoperative dysparania. Neither before or after the POP surgery, there are no data involving sexual dysfunction in Turkish POP patients. Burrows et all showed that about one third of POP patients complain of sexual dysfunction, including dyspareunia in their study. ${ }^{12}$

Another important complication of POP surgery was the mesh exposure. It was detected in 2 vault prolapse patients $(0.84 \%)$, and one of them $(0.42 \%)$ was removed with lochal anesthesia. A Cochrane review reported the rate of mesh extrusion was $11.4 \%$ and $6.8 \%$ of them needed a surgical intervention. ${ }^{13}$ The surgical intervention rate for mesh extrusion reported in another study was $2.6 \% .{ }^{7}$ When uterus preserved and the mesh fixed over the vesicovaginal fasia,in terms of mesh erosion, the risk is lower than when it fixed drectly over vaginal wault.Our mesh extrusion rate is lower according to the literatüre because of more than half of the patients had not hysterectomy before. Among 237 patients $10.5 \%$ of them had hysterectomy and $\% 8$ of them had mesh extrusion. On the other hand,our study groups low rate of sexuel activity may provide less mechanical stress to mesh over the vagina. It is crucial to say that the sexual function of our Turkısh POP patients increased after LLS surgery. The number of sexuel active before surgery is $13(5.4 \%)$; after surgery is $18(7.5 \%)$. So, additional prospective studies with much more patients is needed. Vieillefosse et all reported that up to $50 \%$ of patients, lumbar pain has been described following mesh fixation at the sacrum. ${ }^{14}$ In this study our data on side pain was only $13(5.5 \%)$ patients. If compared LLS with socrohysteropexy,the high lumbar pain rate can be caused by the challenging dissection of the promontorium area, the passage of significant hypogastric nerve plexus and the mesh placed between the sacrum and the vagina is shortened and stretched over time.

In a comparison of the FSFI scores of the 61 patients who completed the questionnaire the arousal score and total score presented considerable improvements after surgery (Table 4). It might be difficult to conclude that the sexual function of our Turkısh POP patients improved after LLS surgery due to the very small number of patients who might have been sexually active (13 patients in before surgery and 14 patients in after surgery), and a further large-scale prospective study is needed. The strengths of this study is regard to the clinical follow-up as a consecutive series of patients operated with a standardized technique by a single surgeon and used the same type mesh. Conversely retrospective design, short-term follow up period, fewer patients are limitations of the study.

\section{Conclusion}

Independent of age Turkish patients with POP presented low rate sexual activity. LLS technic was found to be an effective treatment for POP. Good objective and subjective outcomes and low complications rate was recieved especially mesh extrusion and dyspareunia. Also, this is the first study regarding POP patients sexual activity after LLS surgery.

\section{Acknowledgments}

None.

\section{Funding}

None.

\section{Conflicts of interest}

The authors declare there are no conflicts of interest.

\section{References}

1. Olsen A, Smith V, Bergstrom J, et al. Epidemiology of surgically managed pelvic organ prolapse and urinary incontinence. Obstet Gynecol. 1997;89:501-506.

2. Nygaard I, Barber MD, Burgio KL, et al. Prevalence of symptomatic pelvic floor disorders in US women. JAMA. 2008;300(11):1311-1316.

3. Slieker-ten Hove MCP, Pool-Goudzwaard AL, Eijkemans MJC, et al. The prevalence of pelvic organ prolapse symptoms and signs and their relation with bladder and bowel disorders in a general female population. Int Urogynecol J Pelvic Floor Dysfunct. 2009;20(9):1037-1045.

4. Wu JM, Matthews CA, Conover MM, et al. Lifetime risk of stress urinary incontinence or pelvic organ prolapse surgery. Obstet Gynecol. 2014;123(6):1201-1206. 
5. Digesu GA, Chaliha C, Salvatore S, et al. The relationship of vaginal prolapse severity to symptoms and quality of life. BJOG. 2005;112(7):971-976

6. Bump RC, Mattiasson A, Bø K, et al. The standardization of terminology of female pelvic organ prolapse and pelvic floor dysfunction. Am J Obstet Gynecol. 1996;175(1):10-17.

7. Dubuisson JB, Veit-Rubin N, Wenger JM, et al. Laparoscopic lateral suspension, another way to treat genital prolapse. Gynecol Obstet Fertil Senol. 2017;45(1):32-36.

8. Maher C, Feiner B, Baessler K, et al. Surgery for women with apical vaginal prolapse. Cochrane Database Syst Rev. 2016;10:CD012376.

9. Abdel-Fattah M, Familusi A, Fielding S, et al. Primary and repeat surgical treatment for female pelvic organ prolapse and incontinence in parous women in the UK: a register linkage study. BMJ Open. 2011;1(2):e000206
10. Takahashi M, Inokuchi $\mathrm{T}$, Watanabe $\mathrm{C}$, et al. The female sexual function index (FSFI): development of a Japanese version. J Sex Med. 2011;8(8):2246-2254.

11. Takahashi S, Obinata D, Sakuma T et al. Tension-free vaginal mesh procedure for pelvic organ prolapse: a single-center experience of 310 cases with 1-year follow up. Int J Urol. 2010;17(4):353-358.

12. Burrows LJ, Meyn LA, Walters MD, et al. Pelvic symptoms in women with pelvic organ prolapse. Obstet Gynecol. 2004;1045 Pt 1):982-988.

13. Rosen R, Brown C, Heiman J, et al. The female sexual function index (FSFI): a multidimensional self-report instrument for the assessment of female sexual function. J Sex Marital Ther. 2000;26:191-208.

14. Vieillefosse S, Thubert T, Dache A, et al. Satisfaction, quality of life and lumbar pain following laparoscopic sacrocolpopexy: suture vs. tackers. Eur J Obstet Gynecol Reprod Biol. 2015;187:51-56. 